\title{
Approach to Modeling Boundary Layer Ingestion using a Fully Coupled Propulsion-RANS Model
}

\author{
Justin S. Gray,* \\ NASA Glenn Research Center, Propulsion Systems Analysis Branch, Cleveland OH \\ Charles A. Mader ${ }^{\dagger}$, Gaetan K.W. Kenway ${ }^{\dagger}$, Joaquim R. R. A. Martins ${ }^{\dagger \ddagger}$ \\ University of Michigan, Department of Aerospace Engineering, Ann Arbor MI
}

\begin{abstract}
Airframe-propulsion integration concepts that use boundary layer ingestion have the potential to reduce aircraft fuel burn. One concept that has been recently explored is NASA's Starc-ABL aircraft configuration, which offers the potential for $12 \%$ mission fuel burn reduction by using a turbo-electric propulsion system with an aft-mounted electrically driven boundary layer ingestion propulsor. This large potential for improved performance motivates a more detailed study of the boundary layer ingestion propulsor design, but to date, analyses of boundary layer ingestion have used uncoupled methods. These methods account for only aerodynamic effects on the propulsion system or propulsion system effects on the aerodynamics, but not both simultaneously. This work presents a new approach for building fully coupled propulsive-aerodynamic models of boundary layer ingestion propulsion systems. A 1D thermodynamic cycle analysis is coupled to a RANS simulation to model the Starc-ABL aft propulsor at a cruise condition and the effects variation in propulsor design on performance are examined. The results indicates that both propulsion and aerodynamic effects contribute equally toward the overall performance and that the fully coupled model yields substantially different results compared to uncoupled. The most significant finding is that boundary layer ingestion, while offering substantial fuel burn savings, introduces throttle dependent aerodynamics effects that need to be accounted for. This work represents a first step toward the multidisciplinary design optimization of boundary layer ingestion propulsion systems.
\end{abstract}

\section{Nomenclature}

()$^{\text {fe }} \quad$ quantity computed at the fan-exit boundary

()$^{\mathrm{ff}} \quad$ quantity computed at the fan-face boundary

()$^{\text {prop }}$ quantity computed from the propulsion model

()$_{\infty} \quad$ free stream quantity

$\delta \quad$ boundary layer height

$\dot{m} \quad$ mass flow rate

$\eta_{a} \quad$ adiabatic efficiency

$\eta_{p} \quad$ propulsive efficiency

$\mathcal{R}$ residual function

$\rho \quad$ air density

$\mathrm{A}_{\text {ref }} \quad$ reference wing area

$C_{F-\text { fuse }}$ force coefficient for forces generated by the fuselage

$C_{F-\text { prop }}$ force coefficient for forces generated by the propulsor

$C_{F-x} \quad$ net force coefficient

$d_{\text {nac }} \quad$ nacelle outer diameter

\footnotetext{
*Aerospace Engineer, NASA Glenn Research Center, Propulsion Systems Analysis Branch, AIAA Member

${ }^{\dagger}$ Research Investigator, Department of Aerospace Engineering, AIAA Senior Member

${ }^{\ddagger}$ Professor, University of Michigan, Department of Aerospace Engineering, AIAA Associate Fellow
} 


$\begin{array}{ll}F_{\text {fuse }} & \text { force integrated over the fuselage } \\ F_{\text {prop }} & \text { force integrated over the propulsor } \\ F_{x} & \text { net force integrated over the body } \\ P_{s} & \text { static pressure } \\ P_{t} & \text { total pressure } \\ T_{t} & \text { total temperature } \\ V & \text { air speed }\end{array}$

FPR fan pressure ratio

\section{Introduction}

Although boundary layer ingestion (BLI), or wake ingestion, has been well studied for marine applications since the 1960s [1, 2, 3] it has not yet seen wide-spread adoption in aircraft applications. However, recent studies have considered several new BLI-based aircraft configurations that could offer a reduction in aircraft fuel burn between $5 \%$ and $12 \%[4,5,6]$. This dramatic reduction in fuel burn is achieved via tight integration of the propulsion system and airframe aerodynamics, but actually realizing such large performance gains requires modifying the aircraft design process to account for this integration. Traditionally, in aircraft design, the airframe and the propulsion system are designed separately and then the engine sizing is managed using simple thrust scaling. This works when the propulsion system is placed in free-stream air, away from the aerodynamic influence of the airframe, and it is reasonable to assume that small changes to either system do not affect the other.

BLI systems invalidate this assumption by design. In 1947, the first theoretical study of BLI noted that it would introduce a significant dependence between aircraft drag and engine airflow [7]. Decades later Smith and Robers [8] quantified this interaction from the propulsion system perspective, by relating the propulsive efficiency to a set of nondimensional viscous wake parameters. They found that the overall propulsive efficiency can rise above one in some cases. These efforts highlights the primary challenge of modeling aircraft mission performance in the presence of BLI: the aerodynamic models must be a function of propulsion mass flow, and the propulsion models must be a function of the the boundary layer profile. Drela [9] proposed a control volume approach to the aerodynamic bookkeeping as a way to side-step this issue. Another approach for a more unified bookkeeping is to use the exergy concept to quantify the overall efficiency $[10,11]$.

Despite addressing the thrust and drag accounting problem, these methods require that some level of propulsionaerodynamic coupling is modeled to capture the multidisciplinary interactions that impact on aircraft performance. A number of studies considered the effects of BLI from a quasi-single disciplinary perspective that only captures part of the coupling. Hardin et al. [12] analyzed the effect of the boundary layer velocity profile on fan performance for a traditional turbofan propulsion system, but did not pass the propulsion data back into the aerodynamics to capture the drag effects. Kim and Liou [13, 14] performed a series of aerodynamic shape optimizations on NASA's Hybrid Wing Body (HWB) with BLI propulsion systems using powered boundary conditions, but did not consider propulsion design variables. Blumenthal et al. [15] analyzed a tail cone thruster on the Common Research Model geometry using an actuator disk approach and found that shape optimization of the tail cone offered performance improvements over the baseline design, but their work kept the diameter of and pressure change across the actuator disk constant, which amounted to a fixed propulsion model. There have also been a number of propulsion-centric studies on BLI propulsion systems, but these did not consider the changes to the flow field due to the presence of the engine [5, 16, 17, 4]. All these studies are motivated by the BLI benefits arising from interdisciplinary interactions, but they make assumptions to partially decouple the analyses to make the modeling easier.

Fully coupling the propulsion and aerodynamic analyses presents significant implementation challenges. The propulsion system can be modeled using relatively inexpensive 1D thermodynamic analyses that work with scalar values to represent bulk fluid properties. To capture viscous affects on complex 3D geometries, the aerodynamics modeling requires a RANS simulation. Therefore, a coupling method must be developed to exchange data between the propulsive and aerodynamic models. The need to include RANS models in the design process strongly suggests the need to include shape optimization as part of the design process, since the aerodynamic performance is sensitive to small geometry changes. RANS shape optimization with respect to large numbers of geometry variables requires the use of gradient-based optimization with adjoint analytic derivatives, so a CFD tool that provides an adjoint is required. Due to the propulsive-aerodynamic coupling, the aerodynamic adjoint alone is not sufficient. A fully coupled analysis 
requires a coupled-adjoint in order to consider both propulsion and airframe shape design variables. This means that the propulsion model must also have a correspondent adjoint solver. The coupled adjoint approach, originally developed by Martins et al. [18, 19], is well established for aerostructural applications [20, 21], but has not yet been extended to aero-propulsive applications.

The above challenges have prevented prior work on propulsion-airframe integration from considering the fully coupled system. The ultimate goal of this research is to address these difficulties and perform a Multidisciplinary Design Optimization (MDO) on NASA's Starc-ABL configuration using a fully coupled propulsive-aerodynamic model. However, before we perform design optimization, we must develop the coupled model. Thus, this paper presents the necessary first step: the development of a new coupling approach for propulsive-aerodynamic applications and an associated implementation built in the OpenMDAO framework [22]. A design study was performed to quantify the effects of fan pressure ratio (FPR) on the overall performance of the system. The results show that there is a strong interaction between the propulsion and aerodynamics disciplines that strongly impacts the design of BLI propulsion systems. By analyzing the coupled performance, we demonstrate that both aerodynamic and propulsive effects are equally significant. Furthermore, we show that an uncoupled analysis method misses key multidisciplinary interactions that could cause aerodynamic effects such as throttle dependent lift, drag, and pitching moment.

\section{The Starc-ABL Aircraft Configuration}

In 2016, NASA developed a new aircraft configuration called the Starc-ABL — single-aisle turbo-electric aircraft with an aft boundary layer propulsor - that incorporated BLI into an otherwise traditional airframe layout by adding an aft-mounted propulsor. This aircraft is designed to cruise at Mach 0.72 and 37,000 ft with a wing area of $1400 \mathrm{ft}^{2}$. A rendering of the aircraft configuration is shown in Figure 1. Starc-ABL uses a turbo-electric propulsion system with wing-mounted turbofan engines that also include electric generators to provide power to an electric motor powering the BLI propulsor.

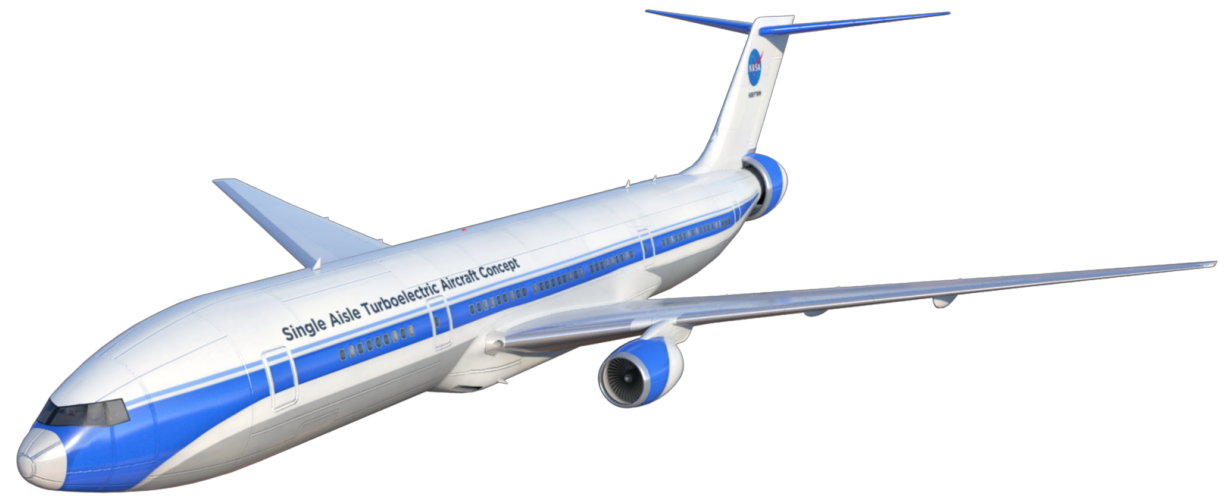

Figure 1: NASA's Starc-ABL aircraft concept, incorporating a hybrid electric propulsion system and aft-mounted BLI propulsor [4]

In their initial design study, Welstead and Felder [4] conclude that this configuration could offer up to a $12 \%$ reduction in fuel burn compared the same configuration with a more traditional propulsion system. For that study, the authors assume that the BLI propulsor would always run with a fixed power input of 3,500 hp, regardless of the flight speed, altitude, or throttle setting of the engine (except at very low throttle settings, where the power requirement was relaxed). Their propulsor had a FPR of 1.2, and an adiabatic efficiency of 95.6\%.

The unique turbo-electric propulsion system lapses differently compared to a traditional under-wing engine configuration. The fixed shaft power input to the BLI propulsor causes it to lapse much less than if it were powered by a traditional gas turbine. The power for the aft propulsor is produced by generators integrated into the under-wing engines. The generators add an additional load to the the low speed spool and cause the engines to lapse more quickly than normal. This means that at low altitudes and speeds — such as take-off — the BLI propulsor does not provide as significant a portion of the overall thrust. However, as the aircraft climbs and increases speed, BLI propulsor begins to contribute a larger share of the overall thrust. According to Welstead and Felder [4], the BLI propulsor provides only $20 \%$ of the overall thrust during take-off, but towards the end of the climb it provides $44 \%$. This means that the performance of the propulsor is much more important at cruise than it is during take-off and climb.

The large share of thrust generated by the BLI propulsor at cruise is ultimately what enables the significant mission fuel burn reductions of this configuration. Hence the design of that propulsor is vitally important to the overall 
performance and viability of the concept, but as previously explained, it is also one of the most challenging aspects to address because of the coupling between the propulsion and aerodynamics analyses.

In this work, we begin a fully coupled detailed study of the BLI propulsor by considering a simplified geometry of an isolated axisymmetric fuselage at zero angle of attack. Although this simplified geometry cannot be considered an aircraft configuration since it does not provide any lift and is not stable, this represents the simplest case where we can capture the primary interdisciplinary interactions for a BLI propulsor on the Starc-ABL configuration. A profile view of the simplified configuration, along with key dimensions are shown in Figure 2.
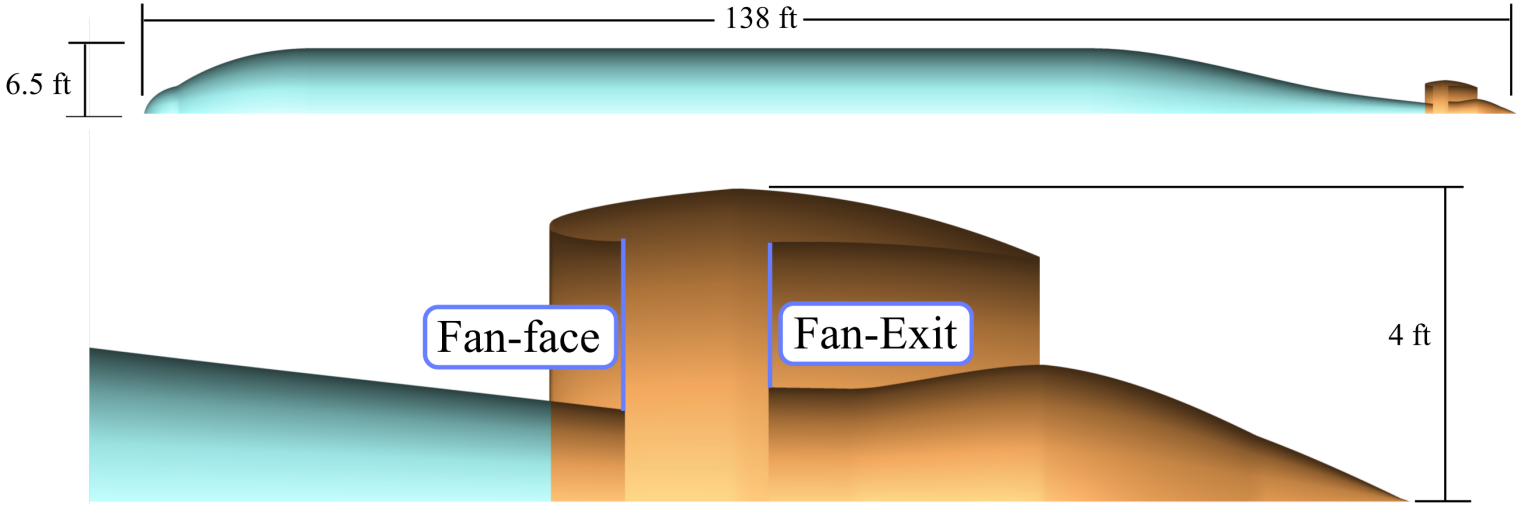

Figure 2: Axisymmetric fuselage with aft mounted BLI propulsor representing a simplified Starc-ABL configuration. For force accounting, the blue section is considered to be fuselage and the orange section is considered to be propulsor.

\section{A. Propulsion Model}

The BLI propulsor is modeled using the 1D thermodynamic cycle analysis tool pyCycle [23, 24]. pyCycle, which is developed using OpenMDAO, allows for flexible modeling of propulsion systems by providing a library of different cycle elements (inlet, compressor, combustor, nozzle, duct) that can be combined to model a specific propulsion system. pyCycle also provides adjoint analytic derivatives for its computations, which will enable optimization with a fully coupled propulsive-aerodynamic adjoint in future work.

The propulsor model in the present work is comprised of a single compressor representing the fan. The model inputs are FPR, and total temperature and pressure at the fan-face. The outputs are the total temperature and pressure at the fan exit, and the mass flow rate the fan can sustain for a fixed shaft power of 3,500 hp. Welstead and Felder selected 3,500 hp in their original work by sizing their propulsor to capture about $70 \%$ of reduced speed air. Their motivation behind this choice was based on an analysis indicating that that increasing the propulsor diameter beyond that level was generating only marginal gains. For this work that assumption was retained, although future work will allow shaft power to vary.

The fan adiabatic efficiency $\left(\eta_{a}\right)$ is computed as a function of FPR. At a lower FPR less flow turning is required and hence a higher adiabatic efficiency can be achieved. We assume the following linear relationship between $\eta_{a}$ and FPR,

$$
\eta_{a}=1.066-0.0866 \mathrm{FPR}
$$

which matches the assumptions made in Welstead and Felder's work.

\section{B. BLI Aerodynamic Model}

The aerodynamic model uses the RANS solver ADflow [20, 25], which provides an efficient viscous adjoint and an in-memory interface to Python that greatly simplifies the integration with OpenMDAO. The axisymmetric geometry allows for a small structured, multiblock mesh with 170,000 cells. On a desktop computer with $3.6 \mathrm{GHz}, 4$ core, 
Intel core-I7 processor and $8 \mathrm{~GB}$ of memory this mesh can be converged 10 orders of magnitude in approximately 2 minutes.

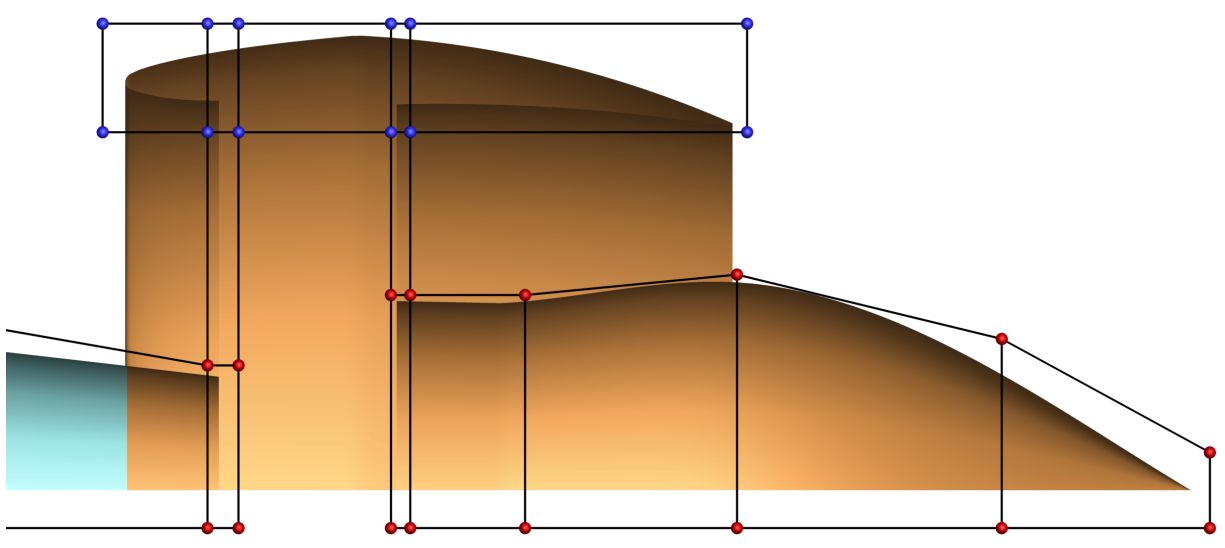

Figure 3: FFD block layout defining the geometry parametrization. Blue nodes define the FFD boxes that move to allow propulsor scaling. Red nodes are held fixed to maintain the fuselage and nozzle plug geometry.

The initial geometry for this model was designed to be similar to a Boeing 737-900 in fuselage length and diameter. Free-form deformation (FFD) was used to parameterize the BLI propulsor diameter. This was accomplished by defining an FFD that rigidly translates the top of the nacelle up or down to scale the propulsor. Figure 3 shows the layout of the FFD boxes that parameterize the geometry, where the blue nodes are the corners of the boxes that translate up and down to scale the nacelle diameter. The red nodes were held fixed to maintain the fuselage and nozzle plug geometry. In future work, additional control points can be added, and both red and blue nodes could be varied to achieve more detailed shape control.

The propulsion related inputs are the diameter of the nacelle $\left(d_{\text {nac }}\right)$ and the two boundary conditions that account for the fan: fan-face and fan-exit, as shown in Figure 2. The fan-face is an outflow boundary condition (flow leaving the CFD domain) where the static pressure is specified. The fan-exit is an inflow boundary condition, where the total pressure and temperature are specified. The model outputs integrated forces on the whole body, $F_{x}$. The model also outputs forces broken down into fuselage $\left(F_{\text {fuse }}\right)$ and propulsor $\left(F_{\text {prop }}\right)$ components. $F_{\text {fuse }}$ is computed as the net force integrated over the blue surfaces in Figure 2. $F_{\text {prop }}$ is computed as the net force integrated over the orange surfaces in Figure 2. Traditionally, $F_{\text {fuse }}$ would be called "drag" and $F_{\text {prop }}$ would be called "thrust". However, for a BLI configuration, thrust and drag are no longer independent quantities and therefore cannot be neatly separated. Thus, we use this force naming approach to draw a clear distinction and highlight the coupled nature of the forces.

The aerodynamic model also outputs the integrated mass flow rates across both the fan-face and fan-exit boundary conditions and the mass-averaged total properties at the fan-face boundary conditions. Taking a mass-average of the total properties, as apposed to an arithmetic-average or area-average, is important for keeping a conservative data transfer between the aerodynamic and propulsion analyses.

\section{Podded Configuration Aerodynamic Model}

To provide a consistent point of reference modeled with the same tools and assumptions as the BLI configuration, we constructed an aerodynamic model emulating a conventional podded configuration. This model consists of two separate simulations: one for the clean fuselage and one for the podded propulsor, as shown in Figure 4.

In this decoupled configuration we can assume that the aerodynamic performance of the fuselage and the propulsor are independent of each other. Using the clean fuselage we can compute a baseline $F_{\text {fuse }}$ and then the $F_{x}$ of the podded configuration can be found by summing $F_{\text {fuse }}$ with the $F_{\text {prop }}$ from a converged simulation of the independent propulsor.

Other than replacing the fuselage with a rounded spinner in the podded propulsor model, the geometry of the nacelle and boundary conditions are identical between the BLI and podded aerodynamic models. This allows the same FFD parameterization to be used for both models, and also means the same coupling scheme is used for both the BLI and podded configurations.

\section{Fully Coupled Model}

Before describing the coupling method used in this work, we clarify what we mean by "coupled" and "uncoupled". We define a coupled model to be one where data is passed between two or more analyses in a reciprocal manner, so 


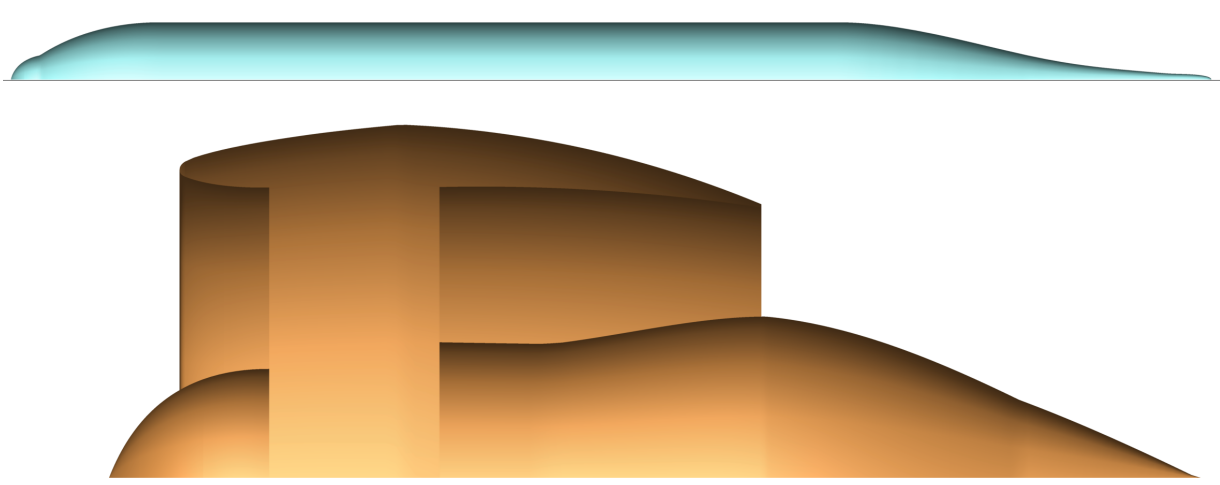

Figure 4: Podded propulsor configuration with clean fuselage (top) and detailed view of the propulsor (bottom).

that some form of iteration (manual or automatic) between the analyses is required to reach physical compatibility. We define uncoupled analysis to be one in which data is passed through sequentially from one model to another, and thus it can be solved with a single pass through the multidisciplinary analysis.

There has been some recent work developing coupled propulsion-aerodynamic models for supersonic applications. Heath et al. [26] manually coupled a RANS analysis to a propulsion model by matching the flow areas and states between the two and using the propulsion model to predict the static pressure for the fan-face boundary condition. This work compared the installed performance and sonic boom for two discrete inlet designs, and the limited number of configurations made manual coupling a reasonable approach. Connolly et al. [27] developed a transient coupled model using an Euler aerodynamic model with 1D gas-dynamics propulsion model for aero-propulso-servo-elasticity applications. They iterate by passing boundary condition values at the interfaces between the two models at every time step to compute state derivatives for the time integration. The work here uses a similar scheme to the one developed by Connolly et al., where thermodynamic states are exchanged by the two models. However, the methods differ in key implementation details. Their implementation integrates the propulsion model directly into the aerodynamic solver, including the coupling terms as additional state variables to be converged with the rest of the simulation. The method used in this work relies on the OpenMDAO framework to manage the multidisciplinary analysis and converge the simulation [22]. This approach is less intrusive, requiring no modifications to either analysis code and allowing for a more complex solver structure.

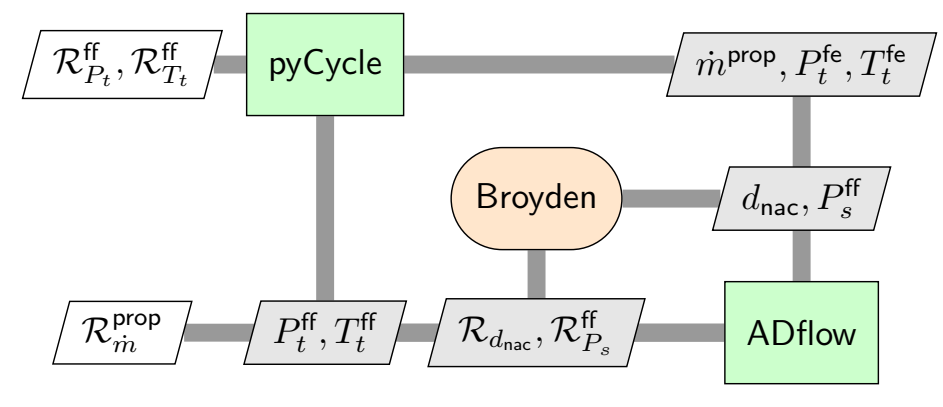

Figure 5: XDSM [28] for the coupled propulsion-aerodynamic analysis.

The fully coupled propulsion-aerodynamic simulation was built with a two-level solver scheme shown in Figure 5, that was used for both the podded and BLI configurations. The top level is converged by a nonlinear Gauss-Seidel solver that manages three residuals defined by the coupling variables: fan-face total pressure $\left(P_{t}^{\mathrm{ff}}\right)$, fan-face total temperature $\left(T_{t}^{\mathrm{ff}}\right)$, and propulsor mass flow rate $\left(\dot{m}^{\text {prop }}\right)$. The inner level is converged using a Broyden solver that handles two additional residuals: $\mathcal{R}_{P_{s}}^{\mathrm{ff}}$ and $\mathcal{R}_{d_{\text {nac }}}$. The mass flow rate between the fan-face and fan-exit boundary conditions is balanced by varying fan-face static pressure $\left(P_{s}^{\mathrm{ff}}\right)$ to converge the $\mathcal{R}_{P_{s}}^{\mathrm{ff}}$ :

$$
\mathcal{R}_{P_{s}}^{\mathrm{ff}}=\dot{m}^{\mathrm{ff}}-\dot{m}^{\mathrm{fe}} .
$$


The mass flow rate between the aerodynamic and propulsion simulations is balanced by varying the nacelle diameter $\left(d_{\text {nac }}\right)$ to converge $\mathcal{R}_{d_{\text {nac }}}^{\text {ff }}$ :

$$
\mathcal{R}_{d_{\text {nac }}}=\dot{m}^{\text {prop }}-\dot{m}^{\text {fe }} .
$$

This last residual is formulated based on the assumption of a fixed input shaft power of 3,500 hp. This provides a convenient way to compare different propulsor designs. Since they all have the same amount of input power, the configuration that provides the highest overall $F_{x}$ is the most efficient.

Note that an alternate reference point is also possible where the power (and hence $\dot{m}^{\text {prop }}$ ) would be varied to drive $F_{x}=0$. In this case, the design with the lowest power input to the propulsor would be the most efficient. Ultimately, this approach was discarded because the Starc-ABL relies on more than just the aft BLI propulsor for its thrust, as it also relies on a pair of under-wing engines. Its not yet clear what the best thrust split between the under-wing engines and BLI propulsor would be, and the fixed-power scheme lends itself more naturally to a problem formulation with a variable thrust split.

\section{Results}

We conducted a trade study by performing a parameter sweep of FPR from 1.2 to 1.35 and then comparing the performance of the podded configuration to that of the BLI configuration. For the podded configuration we assume that changes to the fuselage only affect $F_{\text {fuse }}$, and changes to the propulsion system only affect $F_{\text {prop }}$. With BLI, this assumption is no longer valid and the metric of performance is based on the net-force in the axial direction $\left(F_{x}\right)$ on the combined fuselage-propulsor system. However it is still useful to examine $F_{\text {prop }}$ and $F_{\text {fuse }}$ to understand how these quantities vary across the propulsor design space and how the net performance is achieved.

All results are presented as force coefficients non-dimensionalized as follows:

$$
C_{F}=\frac{2 f}{\rho_{\infty} V_{\infty}^{2} A_{\text {ref }}} .
$$

In our convention the sign of the force, and of the non-dimensional coefficient as well, indicates the direction of action. Positive values represent forward force that cause acceleration. Negative values represent backward force that cause deceleration. The physical values used to non-dimensionalize the forces are listed in Table 1. It is important to note that we retain this convention even when breaking $F_{x}$ into $F_{\text {prop }}$ and $F_{\text {fuse }}$, so it is expected that the $F_{\text {prop }}$ be positive and $F_{\text {fuse }}$ be negative.

Table 1: Reference values for force non-dimensionalization.

\begin{tabular}{cc}
\hline$\rho_{\infty}$ & $0.0008 \mathrm{slug} / \mathrm{ft}^{3}$ \\
$V_{\infty}$ & $707.3 \mathrm{ft} / \mathrm{sec}$ \\
$A_{\text {ref }}$ & $1400 \mathrm{ft}^{2}$ \\
\hline
\end{tabular}

When working with force coefficients it is common refer to "counts of force", which are $10^{4} \times C_{f}$. The results presented below show that the BLI system outperforms the conventional podded configuration by at least 5 force counts across the range of FPR designs considered, and that the aerodynamics and the propulsive improvements both contribute equally to the gains. Thus, a fully coupled analysis is necessary to accurately capture the full BLI effect.

The aerodynamic benefit of the BLI configuration is shown qualitatively in Figure 6, which plots contours of Mach number. The bottom image represents the clean fuselage, the middle one is a BLI configuration with a FPR of 1.35, and the top one is a BLI configuration with a FPR of 1.2. As the FPR is reduced, for the same input power to the fan, the mass flow rate increases, requiring a larger nacelle. The diameter of the nacelle has a strong influence on the height of the boundary layer on the aft fuselage. The largest nacelle, for the FPR of 1.2, also has the thickest boundary layer. The boundary layer heights $(\delta)$ measured at the nacelle lip for the three configurations are listed in Table 2 . The boundary layer grows $15 \%$ between the podded configuration and the BLI configuration with a FPR of 1.35 , while for the FPR 1.2 design it grows by $25 \%$. This variation in the boundary layer profile as a function of the nacelle design demonstrates a sensitivity of the aerodynamics to the propulsion design variable (FPR).

\section{A. Net force as a function of FPR}

The clean fuselage produces a net force of $F_{x}=F_{\text {prop }}-2,331 \mathrm{lbs}$. For the BLI configuration, the net force was computed via integration of the pressure, viscous, and momentum forces on the whole body. The comparison of $F_{x}$ 

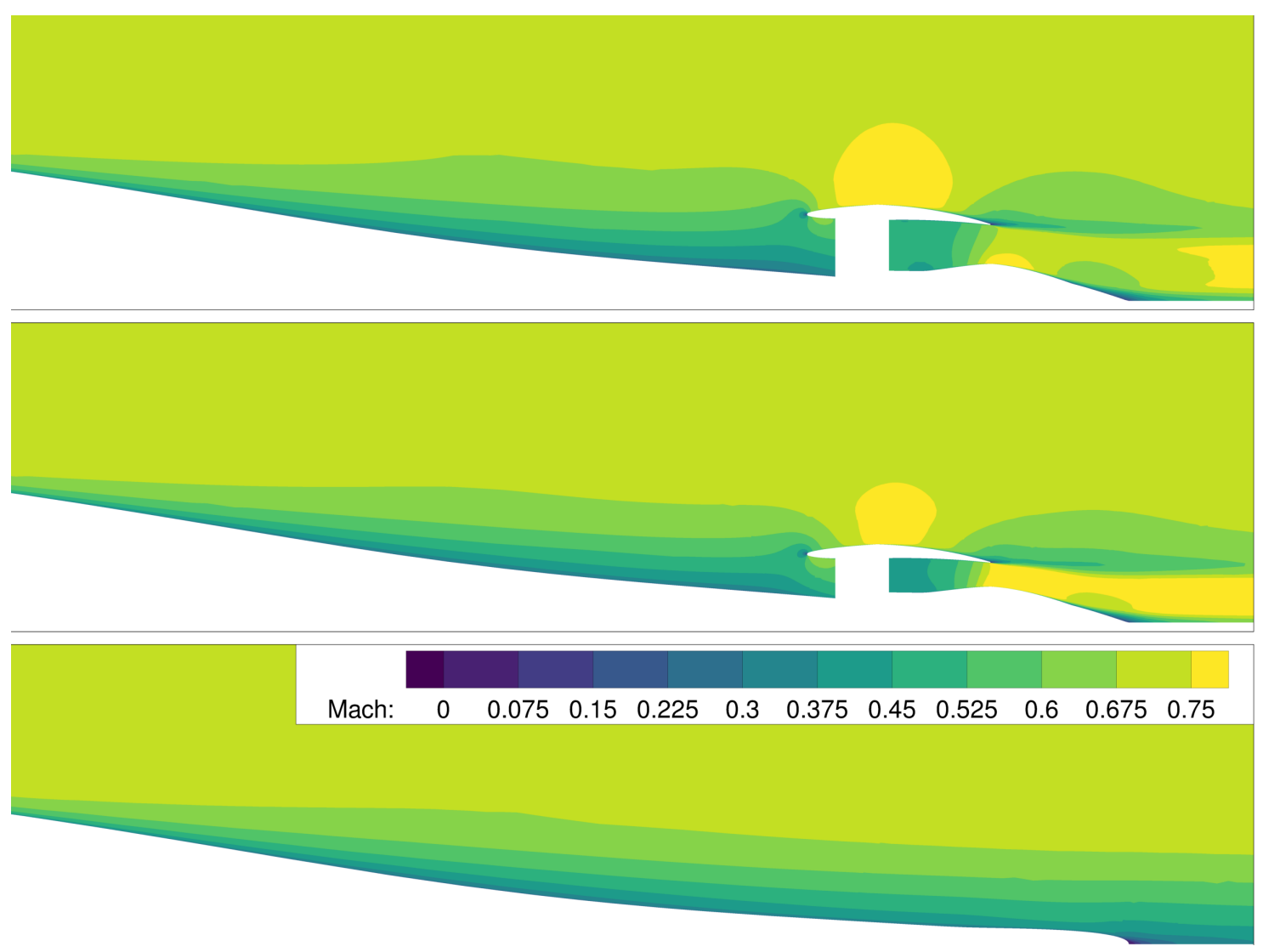

Figure 6: Converged flow solutions for FPR $=1.2$ (top), FPR $=1.35$ (middle), and the clean fuselage (bottom) designs

Table 2: Boundary layer height at the nacelle lip for podded and BLI configurations.

\begin{tabular}{lcc}
\hline configuration & $\delta(\mathrm{ft})$ & $\%$ change \\
\hline podded & 4.0 & \\
BLI, FPR 1.35 & 4.6 & $15 \%$ \\
BLI, FPR 1.2 & 5.0 & $25 \%$ \\
\hline
\end{tabular}

between the podded and BLI configurations is show in Figure 7. The primary conclusion from Figure 7 is that BLI offers an additional 5 to 6 of net force counts. That represents a $33 \%$ improvement in performance relative to the conventional podded configuration. The data also shows two key trends: first, for the podded configuration net force is insensitive to FPR; second, the BLI configuration clearly performs better for lower FPR designs. We can gain further insight into these trends by breaking $C_{F-x}$ down into $C_{F \text {-fuse }}$ and $C_{F \text {-prop }}$ components to look at the contributions from each discipline.

\section{B. Propulsor force as a function of FPR}

Figure 8 shows the variation of the propulsor force with FPR for both podded and BLI configurations. For the podded configuration, where $F_{\text {fuse }}$ is constant, all the variation in $C_{F-x}$ is due to changes in $F_{\text {prop }}$ at different FPR designs. Figure 8 shows that there is only a .2 force count variation in $F_{\text {prop }}$ as a function of FPR for the podded configuration. This result might be surprising if viewed from a purely thermodynamic perspective. Thermodynamics would predict $F_{\text {prop }}$ increasing as FPR is lowered due to higher propulsive efficiency and a higher adiabatic efficiency — defined by Eqn. (1). However, the coupled analysis accounts for both thermodynamic and aerodynamics effects, and while the net thrust does go up, the nacelle drag also increases by nearly as much. Hence, the net effect is a fairly flat response, with slightly better performance towards an FPR of 1.25. The drag increases for lower FPR designs because there is a stronger shock on the outer surface of the nacelle for the larger nacelles, as seen in Figure 9. 


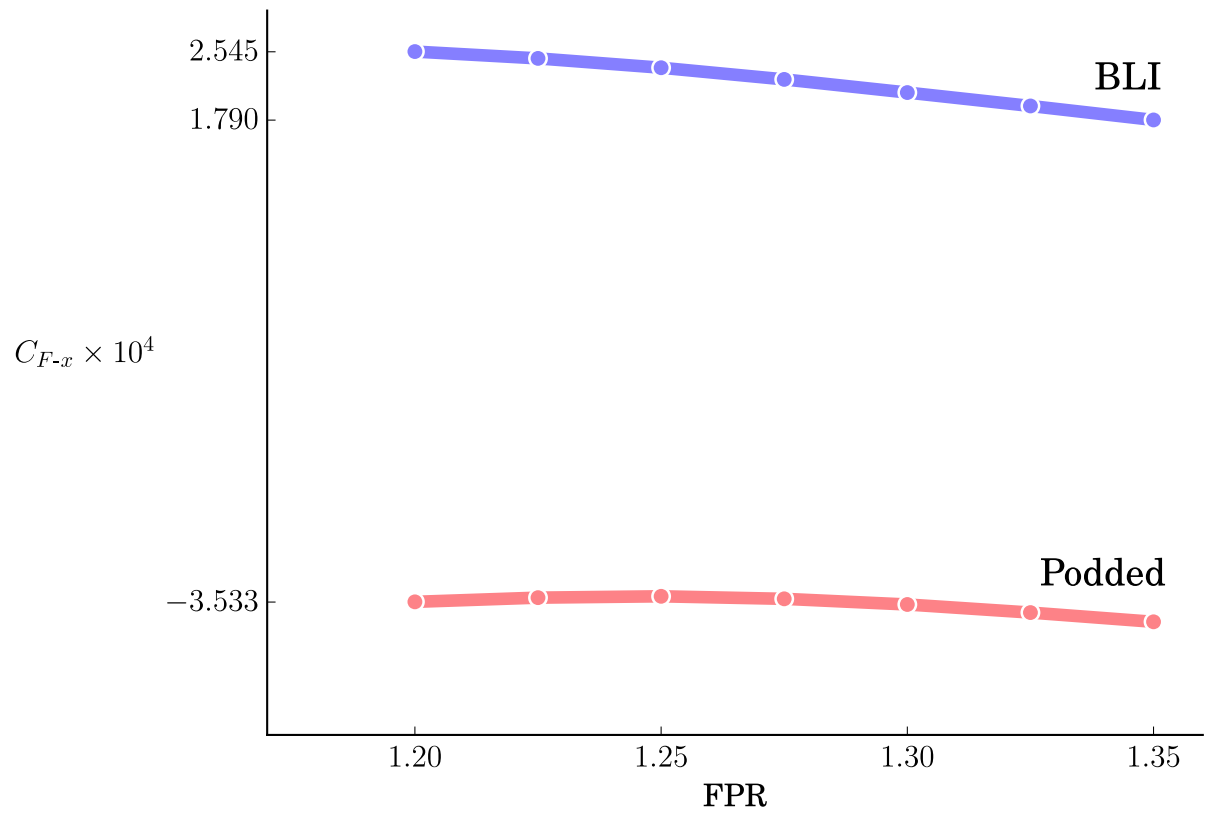

Figure 7: Net force on the body (fuselage and propulsor) as a function of design FPR.

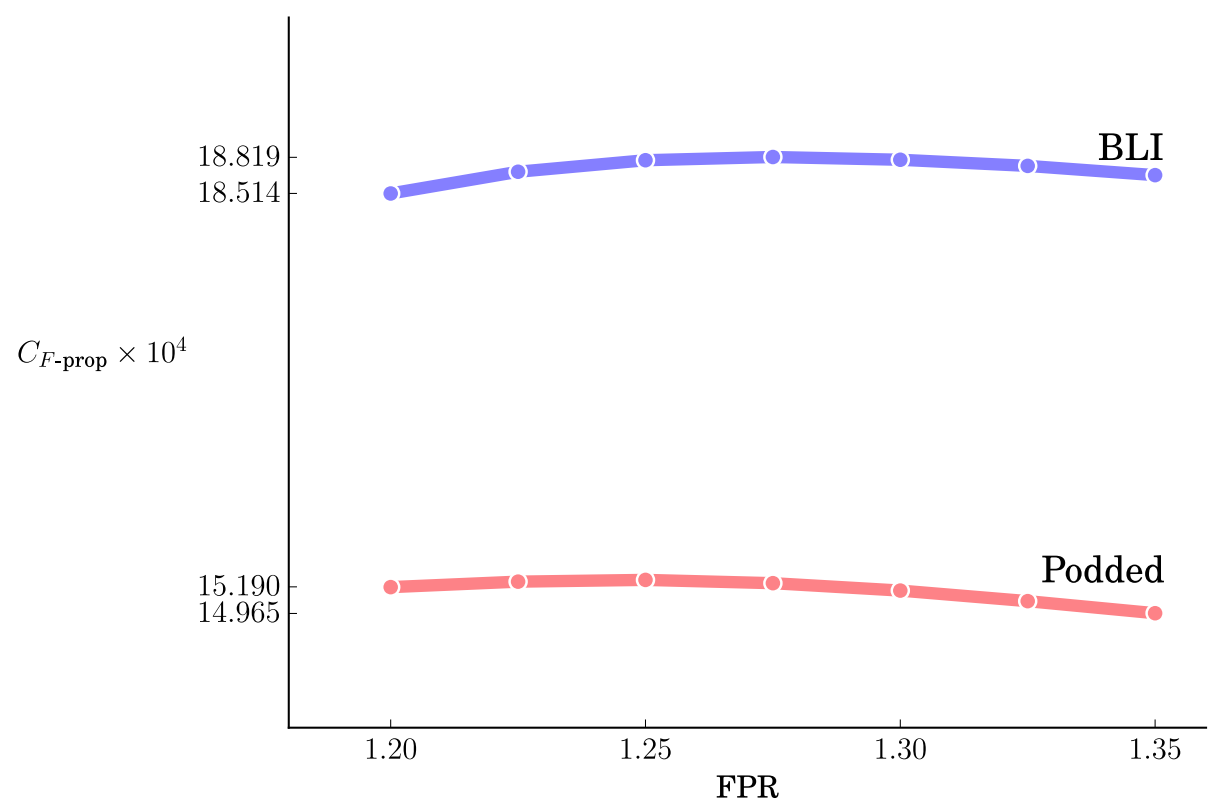

Figure 8: Force integrated over propulsor $\left(C_{F-\text { prop }}\right)$ as a function of design FPR.

The presence of that shock in both configurations indicates that the nacelle geometry is not ideal. In fact, we can see that the same effect is present for the BLI configuration by looking at Figure 6, where the area of high speed flow is larger for the FPR 1.2 configuration on top. Although we would normally expect the larger nacelle for a larger fan to have more drag, it is possible that some of the adverse affect would be mitigated by a better geometry. Future work will use shape optimization to allow for optimal inlet nacelle shaping for each design. However, since this sub-optimal inlet design is used for all the cases presented herein, our performance comparison is valid.

The propulsor force trend for the BLI configuration in Figure 8 shows a different result than the trend in Figure 7. For FPR $=1.35$, the BLI configuration outperforms the podded configuration by 3.9 force counts. For FPR $=1.2$, the difference is 3.3 force counts. Although this variation is small, it is still opposite of the variation of $C_{F_{x}}$ with FPR. 

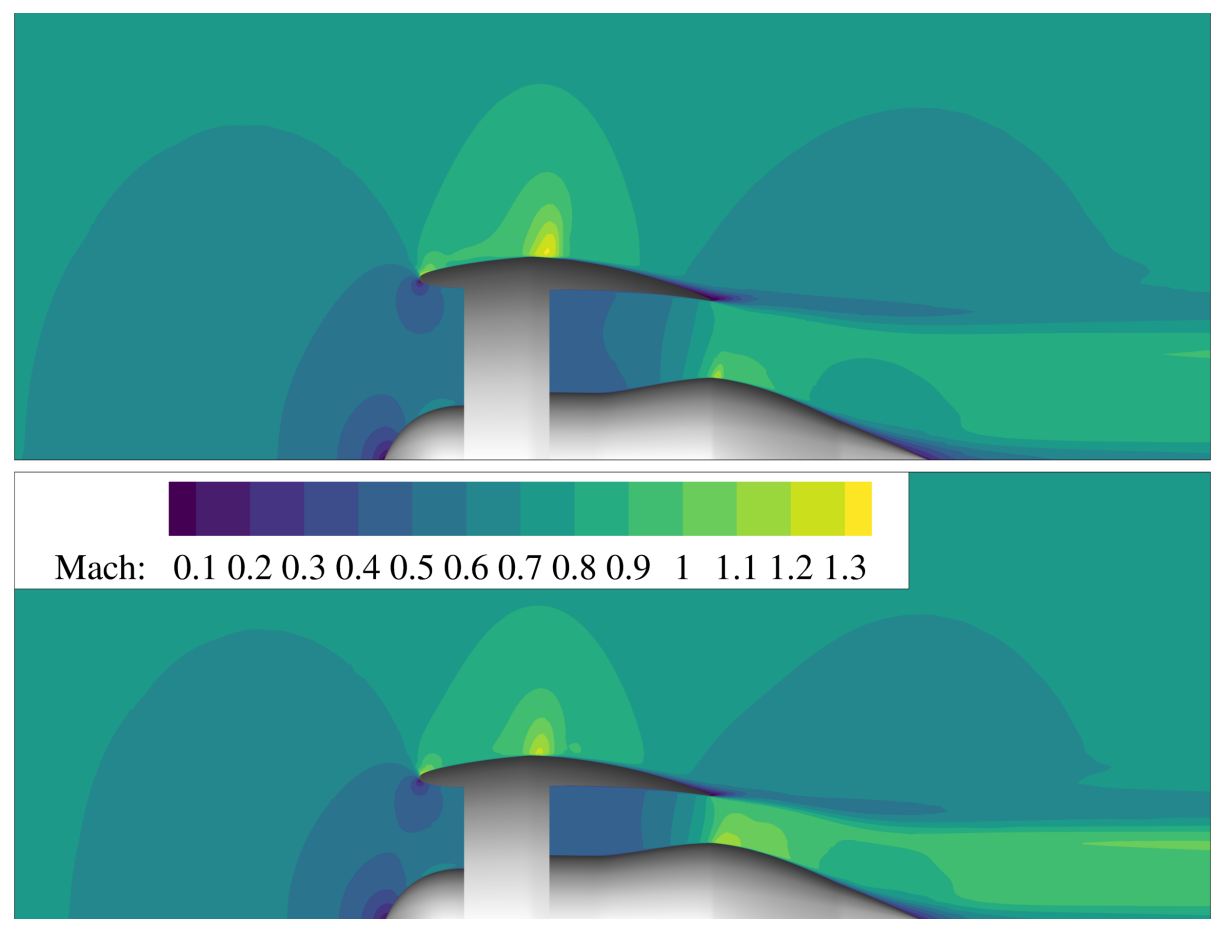

Figure 9: Contours of Mach for the propulsor simulation on the FPR $=1.2$ (top) and FPR $=1.35$ (bottom) designs of the podded configuration.

This BLI trend occurs because as the nacelle grows, the BLI benefit begins to fall off, since more of the engine mass flow is ingested from higher velocity air. Hence, the BLI benefit to the propulsion system becomes less pronounced for lower FPR designs. Additionally, by comparing Figure 7 with Figure 8, we see that only considering the BLI effect on the propulsion analysis would both under-predict the benefit by approximately $40 \%$, and result in a different optimal FPR value.

The difference in magnitude and trend between $C_{F \text {-prop }}$ and $C_{F_{X}}$ highlights the importance of using a fully coupled analysis to study BLI. Another important difference between uncoupled and coupled modeling approaches can be seen by examining the calculation for mass-averaged fan-face total pressure $\left(P_{t}^{\mathrm{ff}}\right)$. For an uncoupled scheme, where we assume that the aerodynamics are unchanged from the podded configuration, $P_{t}^{\mathrm{ff}}$ is computed as follows:

$$
P_{t}^{\mathrm{ff}}\left(d_{\mathrm{nac}}\right)=\frac{\int_{0}^{d_{\mathrm{nac}}} P_{t}(y) \dot{m}(y) d y}{\int_{0}^{d_{\mathrm{nac}}} \dot{m}(y) d y},
$$

where $P_{t}(y)$ is extracted from the boundary layer profile of the clean fuselage in the podded configuration and is independent from any propulsion design variables. This method was used by Welstead and Felder [4] in their study of the Starc-ABL configuration, and by Liu et. al [16] and Laskaridis et al. [17] in their studies of a hybrid wing body turbo-electric distributed propulsion configuration. With the fully coupled analysis $P_{t}(\mathrm{FPR}, y)$ and $\dot{m}(\mathrm{FPR}, y)$ are now a function of both propulsor design and diameter, and hence $P_{t}^{\mathrm{ff}}$ becomes

$$
P_{t}^{\mathrm{ff}}\left(\mathrm{FPR}, d_{\mathrm{nac}}\right)=\frac{\int_{0}^{d_{\text {nac }}} P_{t}(\mathrm{FPR}, y) \dot{m}(\mathrm{FPR}, y) d y}{\int_{0}^{d_{\text {nac }}} \dot{m}(\mathrm{FPR}, y) d y} .
$$

Figure 10 compares mass-averaged $P_{t}^{\mathrm{ff}}$ as a function of FPR between podded configuration and the BLI configuration. The BLI results are computed using both uncoupled - Eqn. (5) - and coupled -Eqn. (6) - methods. For the podded configuration, $P_{t}^{\mathrm{ff}}$ is independent of FPR since the inlet ingests freestream air. The BLI configuration analyzed using the uncoupled method (dashed blue line), does capture the inverse relationship of $P_{t}^{\mathrm{ff}}$ with FPR. However, it over-predicts $P_{t}^{\mathrm{ff}}$ by about 5\% compared to the coupled method (solid blue line). $P_{t}^{\mathrm{ff}}$ has a meaningful impact on propulsor performance. For an electrically driven propulsor, a 5\% lower $P_{t}^{\mathrm{ff}}$ means $5 \%$ lower nozzle pressure ratio, and hence nearly $5 \%$ lower thrust. The effect would be even more pronounced for a BLI design using a conventional turbofan, where the additional total pressure loss would have a direct impact on the overal pressure ratio and thermal 
efficiency of the engine. Thus, accurate performance results require considering the aerodynamic changes caused by the presence of the propulsor.

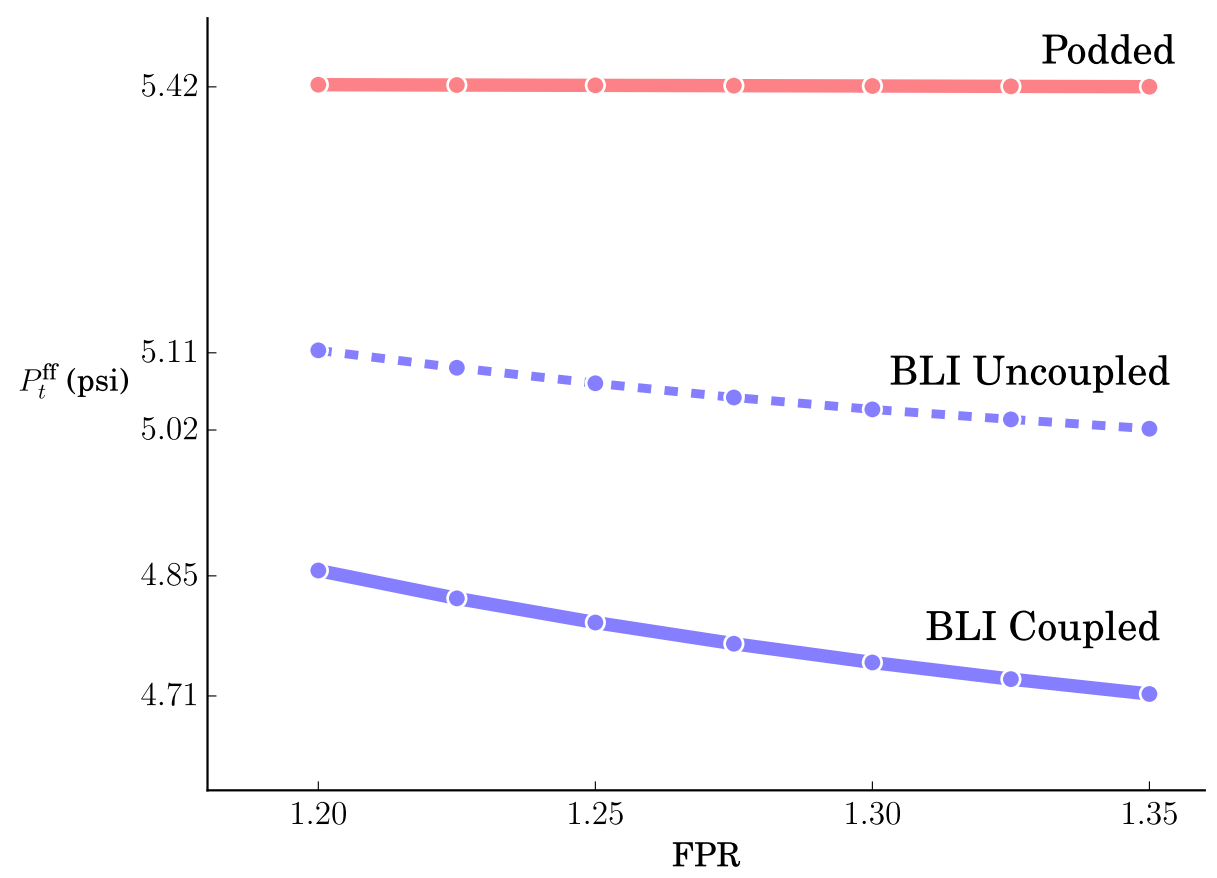

Figure 10: Mass averaged $P_{t}^{\mathrm{ff}}$ as a function of FPR for the podded configuration (pink), the BLI configuration with a fully coupled analysis (solid blue) and a decoupled analysis (dashed blue)

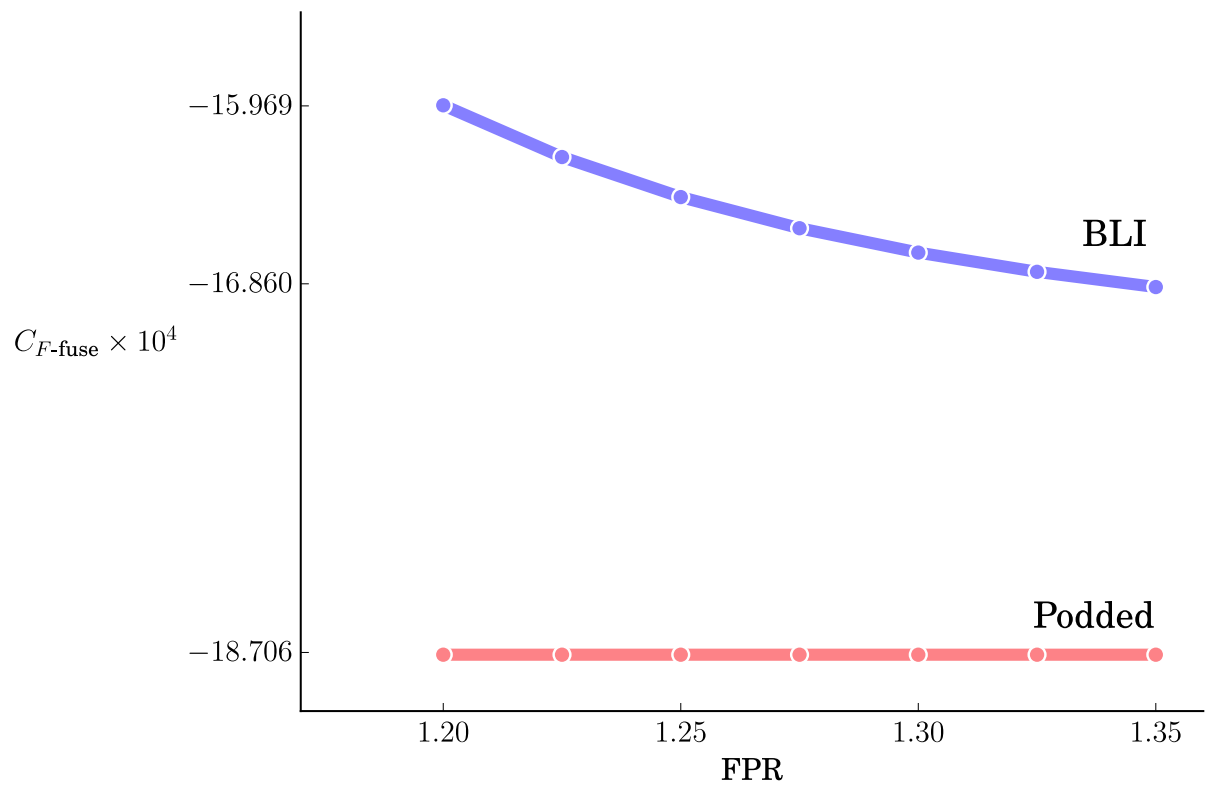

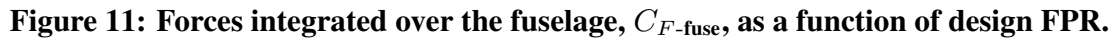

\section{Fuselage force as a function of FPR}

Figure 11 shows the aerodynamic contribution to the overall performance. The podded data is constant by definition, since there is no aerodynamic interaction between the propulsor and the fuselage. The BLI configuration has better $C_{F \text {-fuse }}$ for all designs by at least 1.8 force counts. However, unlike the forces on the propulsor, $C_{F \text {-fuse }}$ for the BLI 
configuration shows strong variation with FPR. From the FPR $=1.35$ design to the FPR $=1.2$ design, the $C_{F \text {-fuse }}$ improves by a further 1.1 counts. The total variation in $C_{F \text {-fuse }}$ is larger than the total variation in $C_{F \text {-prop }}$, indicating that the aerodynamic performance is more sensitive to FPR - a propulsor design variable - than the propulsor performance is.

The sensitivity of the aerodynamics to FPR in the BLI configuration is due to variations in the flow field in the aft fuselage. We can see this effect qualitatively in Figure 6. The actual velocity profiles for the podded configuration, and the BLI configuration for FPRs of 1.35 and 1.2 are shown in Figure 12. In this data we see that the propulsor slows down the flow near the surface and that the effect reaches about $4 \mathrm{ft}$ forward of the fan-face. Also note that the larger propulsor for the FPR $=1.2$ case has a more significant impact on the flow than the smaller propulsor for FPR $=1.35$ case.

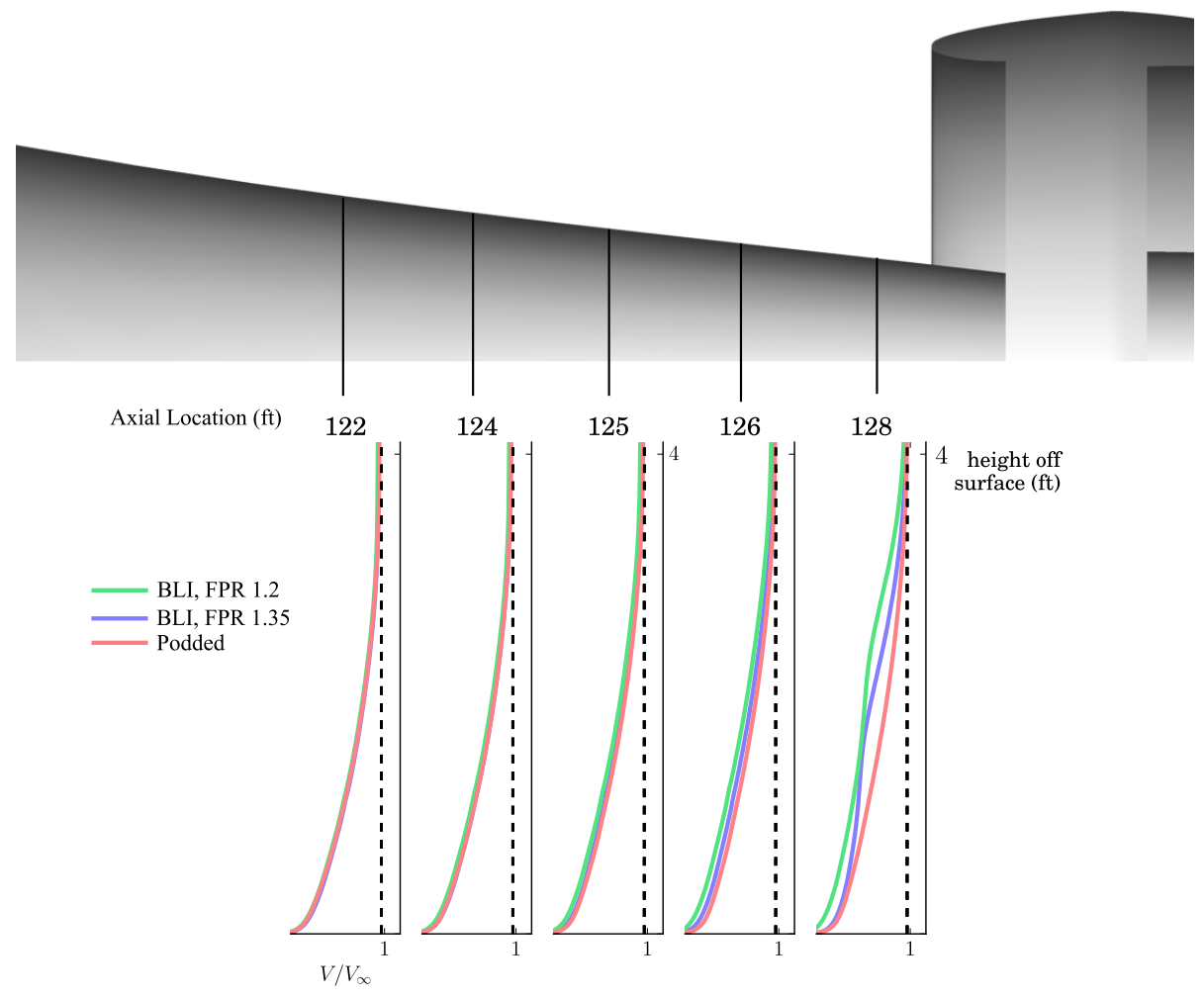

Figure 12: Comparison of boundary layer profiles at the aft fuselage between the podded and BLI configurations.

The boundary layer profiles directly show that the flow on the aft fuselage is influenced by the aft propulsor. These profiles also explain why the coupled analysis predicts a lower $P_{t}^{\mathrm{ff}}$ than the uncoupled analysis, as shown in Figure 10. The presence of the propulsor causes the boundary layer height to grow, an effect that is not captured by the uncoupled analysis. The taller boundary layer yields lower air speeds at the inlet and hence lower the total pressure. The lower flow speeds in the boundary layer also suggest a possible explanation for the reduced $F_{\text {fuse }}$ in the BLI configuration. However, the difference in boundary layer profiles does not propagate very far forward. At $122 \mathrm{ft}$, just $6 \mathrm{ft}$ upstream of the inlet lip, the profiles reverted to being nearly identical.

In order to better understand the aerodynamic cause for the change in $F_{\text {fuse }}$, we examined the static pressure along the surface of the aft fuselage. Figure 13 confirms that there is a significant difference in the pressure along the aft fuselage, but it also shows that the effect reaches farther upstream on the fuselage than the variation in boundary layer profiles. While the difference in pressure is only a about $0.1 \mathrm{psi}$, it is acting on a large base area, and so it has a significant affect. The pressure data in Figure 13 explains the trend for the BLI configuration in Figure 11. As the FPR decreases, and the propulsor increases in diameter, the effect of the higher pressure at the fan-face propagates farther upstream and decreases $F_{\text {fuse }}$ more.

The trends in Figure 13 summarize the most significant finding of this work: the presence of the propulsor in a BLI configuration can have a large impact on the pressure distribution along the aerodynamic surface it is attached to. Furthermore, we can see that the details of that interaction are highly sensitive to changes in the propulsor design 


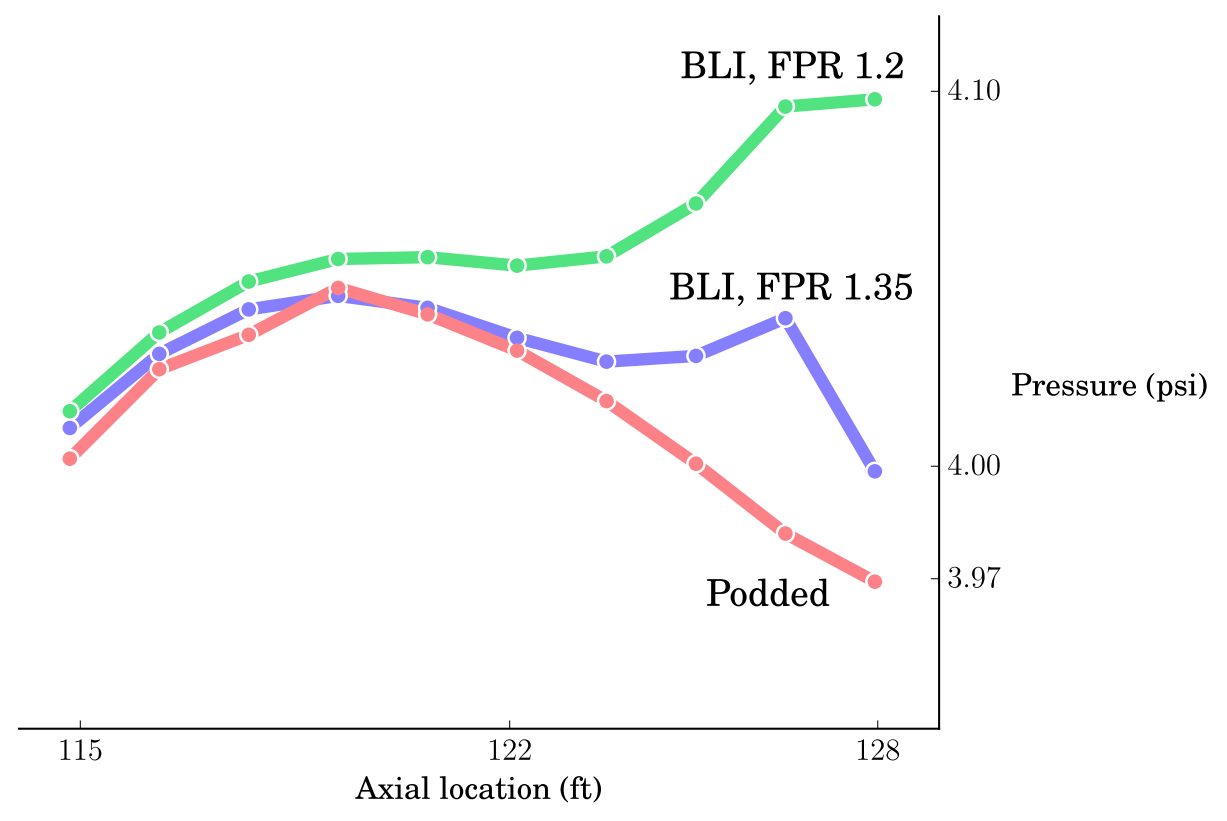

Figure 13: Comparison of surface pressures along the aft fuselage between the podded configuration and BLI configurations for FPR 1.35 and FPR 1.2 cases.

and shaft input power which would affect $P_{s}^{\mathrm{ff}}$. Our results show an inverse relationship between $P_{s}^{\mathrm{ff}}$ and FPR, but that relationship could be altered by varying other aspects of the propulsor design not considered here. For example, $P_{s}^{\mathrm{ff}}$ is a function of both throttle setting and inlet design. Throttling down would increase $P_{s}^{\mathrm{ff}}$, while throttling up would decrease it. Even for a fixed FPR, changes to inlet design would also alter the $P_{s}^{\mathrm{ff}}$. Higher $P_{s}^{\mathrm{ff}}$ — which would reduced drag - could be achieved with larger and more diffusive inlets. However, that would further enlarge the nacelle and nozzle plug, which would decrease the propulsion performance.

\section{Conclusions}

We presented a new approach for building coupled propulsion-aerodynamic analyses of BLI propulsion systems using a 1D cycle model and a RANS aerodynamic model. The approach was implemented in the OpenMDAO framework, using pyCycle and ADflow for the propulsion and aerodynamic analyses, respectively. A simplified version of NASA's Starc-ABL configuration was modeled and a parameter sweep of propulsor FPR from 1.2 to 1.35 was performed to study the coupled performance of the aft-mounted BLI propulsor and characterize the contributions from each discipline. The coupled data shows that BLI offers at least 5 force counts of increased performance relative to the podded configuration across the FPR range. That represents a 33\% increase in thrust relative to the 15 force counts of thrust for the podded configuration, which clearly demonstrates the large potential for improved performance when using BLI propulsion systems in this configuration.

The trends were further analyzed by breaking down the net force down into aerodynamic and propulsive contributions. The breakdown shows that 2 force counts were due aerodynamic benefits and 3 counts we due to propulsion benefits. Thus, we conclude that the BLI configuration results in a performance improvements due to both aerodynamics and propulsion, and each one is equally important. These results demonstrate the need to use a fully coupled model to predict the performance of the BLI configuration. Considering only propulsion or only aerodynamics would ignore half of the overall effect.

The BLI benefit to the propulsion system arises primarily from a decrease in incoming momentum flux (a reduction in ram drag). The thrust data presented here shows that this benefit is only a weak function of FPR because of the offsetting thermodynamic improvements and nacelle drag increases. It is possible that a more refined nacelle geometry would alleviate some of the drag penalties for the lower FPR designs shown here, and change the trends.

The BLI benefit to the aerodynamics was due to a higher static pressure along the aft section of the fuselage caused by static pressure at the fan-face boundary condition. While the propulsion effect was slightly larger in magnitude, the aerodynamic effect was far more sensitive to FPR. This occurred because, with a fixed input shaft power, the FPR was effectively also controlling the nacelle diameter (i.e., a lower FPR means a larger nacelle). The diameter of the 
nacelle had a direct effect on how far upstream the BLI effects propagated and meant that lower FPR designs had a much larger aerodynamic effect. The variation in pressure along the surface of the aft fuselage indicates that BLI propulsion systems can have a strong impact on the pressure distribution of the associated aerodynamic surfaces. For the Starc-ABL configuration, this yields a decrease in drag, but other configurations, such as the MIT-D8 or TurboElectric Distributed Propulsion, could potentially see additional aerodynamic affects. For these aircraft, the variation in the surface pressure caused by the BLI propulsion system could cause not only propulsion-dependent drag effects, but also lift and pitching moments. This means that BLI could ultimately result in throttle-dependent angle of attack and trim settings.

It is clear that the overall performance of the BLI configuration depends heavily on both propulsion and aerodynamic performance and also that each discipline strongly impacts the other. Both propulsion and aerodynamic performance are impacted by design choices from the other discipline. This considered only a single propulsor design variable, FPR. However performance is also likely to be sensitive to other variables, such as fuselage geometry, nacelle geometry, inlet design, and shaft input power. Therefore, to achieve optimal overall performance, a careful balance of aerodynamic and propulsive considerations is required. Future work to refine this concept will use design optimization with a gradient-based algorithm and adjoint analytic derivatives to simultaneously optimize propulsion and aerodynamics design variables.

\section{Acknowledgments}

The authors would like to acknowledge Bret Naylor, Dr. Ken Moore, and the rest of the NASA Glenn OpenMDAO development team, who have spent countless hours building the framework that has enabled this research. We would also like to thank James Felder, of the NASA Glenn Research Center, for lending his time and expertise to many discussions regarding the propulsion system design of the Starc-ABL configuration. We also gratefully acknowledge NASA's Aeronautics Research Mission Directorate Transformational Tools and Technologies (TTT) and the Advanced Air Transport Technologies (AATT) projects for their continued support of this work. 


\section{References}

[1] Betz, A. and Albring, W., "Introduction to the Theory of Flow Machines," ZAMM - Journal of Applied Mathematics and Mechanics / Zeitschrift fr Angewandte Mathematik und Mechanik, Vol. 47, No. 2, 1967, pp. 140-141. doi:10.1002/zamm.19670470224.

[2] Wislicenus, G. F., "Hydrodynamics and Propulsion of Submerged Bodies," Journal of the American Rocket Society, Vol. 30, December 1960, pp. 1140-1148.

[3] Gearhart, W. S. and Henderson, R. E., "Selection of a Propulsor for a Submersible System,” Journal of Aircraft, Vol. 3, No. 1, 1966, pp. 84-90.

[4] Welstead, J. R. and Felder, J. L., "Conceptual Design of a Single-Aisle Turboelectric Commercial Transport with Fuselage Boundary Layer Ingestion,” 54th AIAA Aerospace Sciences Meeting, AIAA 2016-1027, 2016. doi:10.2514/6.2016-1027.

[5] Felder, J. L., Kim, H. D., and Brown, G. V., "Turboelectric Distributed Propulsion Engine Cycle Analysis for Hybrid-WingBody Aircraft," 47th AIAA Aerospace Sciences Meeting including The New Horizons Forum and Aerospace Exposition, AIAA 2009-1132, 2009. doi:10.2514/6.2009-1132.

[6] Daggett, D. L., Kawai, R., , and Friedman, D., "Blended Wing Body Systems Studies: Boundary Layer Ingestion Inlets With Active Flow Control,” Tech. Rep. NASA/CR-2003-212670, NASA Langley Research Center, December 2003.

[7] Smith, A. M. O. and Roberts, H. E., "The Jet Airplane Utilizing Boundary Layer Ingestion for Propulsion," Journal of Aeronautical Sciences, Vol. 14, No. 2, 1947, pp. 97-109.

[8] Smith, L. H., "Wake Ingestion Propulsion Benefit," Journal of Propulsion and Power, Vol. 9, No. 1, February 1993, pp. 74-82. doi:10.2514/6.1991-2007.

[9] Drela, M., "Power Balance in Aerodynamic Flows," AIAA Journal, Vol. 47, No. 7, 2009, pp. 1761-1771. doi:10.2514/1.42409.

[10] Arntz, A., Atinault, O., and Merlen, A., "Exergy-Based Formulation for Aircraft Aeropropulsive Performance Assessment: Theoretical Development," AIAA Journal, Vol. 53, No. 6, 2015, pp. 1627-1639. doi:10.2514/1.J053467.

[11] Arntz, A. and Atinault, O., "Exergy-Based Performance Assessment of a Blended WingBody with Boundary-Layer Ingestion," AIAA Journal, Vol. 53, No. 12, 2015, pp. 3766-3776. doi:10.2514/1.J054072.

[12] Hardin, L., Tillman, G., Sharma, O., Berton, J., and Arend, D., “Aircraft System Study of Boundary Layer Ingesting Propulsion," 48th AIAA/ASME/SAE/ASEE Joint Propulsion Conference and Exhibit, AIAA-2012-2993, 2012. doi:10.2514/6.20123993.

[13] Kim, H. and Liou, M.-S., "Optimal Inlet Shape Design of N2B Hybrid Wing Body Configuration," Proceedings of the 48th AIAAASME/SAE/ASEE Joint Propulsion Conference and Exhibit, AIAA-2012-3917, 2012. doi:10.2514/6.2012-3917.

[14] Kim, H. and Liou, M.-S., "Optimal Shape Design of Mail-Slot Nacelle on N3X Hybrid Wing-Body Configuration,” Proceedings of the 31st AIAA Applied Aerodynamics Conference, AIAA-2013-2413, 2013. doi:10.2514/6.2013-2413.

[15] Blumenthal, B., Elmiligui, A. A., Geiselhart, K., Campbell, R. L., Maughmer, M. D., and Schmitz, S., "Computational Investigation of a Boundary-Layer Ingestion Propulsion System for the Common Research Model," 46th AIAA Fluid Dynamics Conference, Aviation Forum, AIAA 2016-3812, 2016. doi:10.2514/6.2016-3812.

[16] Liu, C., Doulgeris, G., Laskaridis, P., and Singh, R., "Thermal cycle analysis of turboelectric distributed propulsion system with boundary layer ingestion," Aerospace Science and Technology, Vol. 27, No. 1, 2013, pp. 163 - 170. doi:http://dx.doi.org/10.1016/j.ast.2012.08.003.

[17] Laskaridis, P., Pachidis, V., and Pilidis, P., "Opportunities and challenges for distributed propulsion and boundary layer ingestion," Aircraft Engineering and Aerospace Technology, Vol. 86, No. 6, 2014, pp. 451-458. doi:10.1108/AEAT-05-20140067.

[18] Martins, J. R. R. A., Alonso, J. J., and Reuther, J. J., "High-Fidelity Aerostructural Design Optimization of a Supersonic Business Jet," Journal of Aircraft, Vol. 41, No. 3, 2004, pp. 523-530. doi:10.2514/1.11478.

[19] Martins, J. R. R. A., Alonso, J. J., and Reuther, J. J., "A Coupled-Adjoint Sensitivity Analysis Method for High-Fidelity Aero-Structural Design,” Optimization and Engineering, Vol. 6, No. 1, March 2005, pp. 33-62. doi:10.1023/B:OPTE.0000048536.47956.62.

[20] Kenway, G. K. W. and Martins, J. R. R. A., "Multipoint High-fidelity Aerostructural Optimization of a Transport Aircraft Configuration," Journal of Aircraft, Vol. 51, 2014, pp. 144-160. doi:10.2514/1.C032150. 
[21] Liem, R. P., Kenway, G. K. W., and Martins, J. R. R. A., "Multimission Aircraft Fuel Burn Minimization via Multipoint Aerostructural Optimization,” AIAA Journal, Vol. 53, 2015, pp. 104-122. doi:10.2514/1.J052940.

[22] Gray, J. S., Hearn, T. A., Moore, K. T., Hwang, J., Martins, J., and Ning, A., "Automatic Evaluation of Multidisciplinary Derivatives Using a Graph-Based Problem Formulation in OpenMDAO," 15th AIAA/ISSMO Multidisciplinary Analysis and Optimization Conference, American Institute of Aeronautics and Astronautics, August 2014. doi:10.2514/6.2014-2042.

[23] Hearn, D. T., Hendricks, E., Chin, J., Gray, J., and Moore, D. K. T., "Optimization of Turbine Engine Cycle Analysis with Analytic Derivatives," 17th AIAA/ISSMO Multidisciplinary Analysis and Optimization Conference, part of AIAA Aviation 2016 (Washington, DC), 2016. doi:10.2514/6.2016-4297.

[24] Gray, J., Chin, J., Hearn, T., Hendricks, E., Lavelle, T., and Martins, J. R. R. A., “Thermodynamics For Gas Turbine Cycles With Analytic Derivatives in OpenMDAO," 57th AIAA/ASCE/AHS/ASC Structures, Structural Dynamics, and Materials Conference, AIAA SciTech. doi:10.2514/6.2016-0669.

[25] Kenway, G. K. W., Kennedy, G. J., and Martins, J. R. R. A., "Scalable parallel approach for high-fidelity steady-state aeroelastic analysis and adjoint derivative computations," AIAA Journal, Vol. 52, 2014, pp. 935-951. doi:10.2514/1.J052255.

[26] Heath, C. M., Slater, J. W., and Rallabhandi, S. K., "Inlet Trade Study for a Low-Boom Aircraft Demonstrator," Journal of Aircraft, 2016. doi:DOI: 10.2514/1.C034036.

[27] Joseph W. Connolly, Pawel Chwalowski, M. D. S. J.-r. C. W. A. S. J. J. M. and Kopasakis, G., "Towards an Aero-PropulsoServo-Elasticity Analysis of a Commercial Supersonic Transport," 15th Dynamics Specialists Conference, AIAA SciTech Forum, 2016. doi:http://dx.doi.org/10.2514/6.2016-1320.

[28] Lambe, A. B. and Martins, J. R. R. A., "Extensions to the Design Structure Matrix for the Description of Multidisciplinary Design, Analysis, and Optimization Processes," Structural and Multidisciplinary Optimization, Vol. 46, 2012, pp. 273-284. doi:10.1007/s00158-012-0763-y. 